SIR,-Dr C J Ellis (2 April, p 952) supported by Drs E L C Ong and E M Dunbar (30 April, p 1259) recommends quinine for falciparum malaria because of the death of a patient who received chloroquine for prophylaxis and treatment. In $1976^{1}$ and $1978^{2}$ I advised quinine by intravenous infusion in a dose of $5-10 \mathrm{mg} / \mathrm{kg}$ over four hours roughly every 12 hours for severe falciparum malaria. In 1982 I recommended quinine by intravenous infusion in all patients because severe falciparum malaria can appear mild clinically, Since then most falciparum infections at this hospital have been treated with quinine. We do treat most mild infections with the oral quinine and Fansidar regimen, but when in doubt the intravenous route is used.

Since 1977 intravenous quinine in a dose of up to $10 \mathrm{mg} / \mathrm{kg}$ (maximum $500 \mathrm{mg}$ ) at least every 12 hours has caused no severe toxicity in 79 patients. On eight hourly dosing, however, nine out of 22 patients developed severe cinchonism $(p<0 \cdot 01)$ No patients have died of malaria at this hospital since 1978. I recommend that the infusions of quinine should be given about every 12 hours until the patient has greatly improved, when the treatment should be changed to oral quinine for a few doses or Fansidar immediately. Mild cinchonism can occur with 12 hourly dosing, and a flexible response is needed. For example, if the parasitaemia falls rapidly the dose of quinine should be halved or omitted. If the patients are severely jaundiced or in renal failure the dosage interval should usually be 24 hours.

I suggest a therapeutic trial of oral chloroquine as an inpatient treatment in pregnant African women with clinically mild falciparum malaria and parasitaemia of less than $1 \%$ because of the fear (mostly unfounded) that quinine can cause abortion in therapeutic doses and because chloroquine is usually curative in such patients. Intravenous quinine has been successful and nontoxic here in pregnant women with parasitaemia of $2-30 \%$.

Hospital for Tropical Diseases,

ANTHONY HaLI

London NW1 OPE

1 Hall AP. The treatment of malaria. Br Med f 1976;i:323-8. 2 Hall AP. Preventing deaths from malaria. Br Med J 1978;ii:

3 Hall AP. Quinine by intravenous infusion for falciparum malaria BrMed f 1982;285:439.

\section{Sunbed lentigines}

SIR,-Dr H C Williams and colleagues (16 April, p 1097) drew attention to the fact that the emission from ultraviolet $A$ sunbeds includes small amounts of ultraviolet B radiation $(280-315 \mathrm{~nm})$, which may, they think, have been implicated in the induction of the lentigines which they described. This possibility was also discussed by Jones $e t a l,{ }^{1}$ who described a similar case.

Clinical experience in the clearance of psoriasis by ultraviolet $B$ phototherapy indicates that patients do not develop pigmented lesions despite the fact that the cumulative dose of ultraviolet $B$ required is higher than that received by the authors' patient in 50 half hour sessions on an ultraviolet A sunbed (table). On the other hand, the cumulative dose of ultraviolet $\mathrm{A}$ received in

Typical ultraviolet radiation exposure doses

Total dose $\left(\mathrm{J} / \mathrm{cm}^{2}\right)$

Type of exposure

Ultraviolet B Ultraviolet A

Clearance of psoriasis by ultraviolet $B$ phototherapy

Cosmetic tanning, 50 half hour sessions
Ultraviolet lamp

Mercury arc (eg Alpine sunlamp)

this course of cosmetic tanning was about 300 times that received in a course of ultraviolet $B$ phototherapy. In the case described therefore the responsible radiation probably lay within the ultraviolet $A$ waveband.

We were surprised to read the statement that ultraviolet A sunbeds were ineffective in producing a satisfactory tan. In a survey of over 1000 people using ultraviolet A sunbeds for cosmetic tanning 98\% reported some tanning and 38\% reported obtaining a deep tan.

Regional Medical Physics Department,

B L DIFFEY

Respital,

Department of Dermatology,

Royal Victoria Infirmary

Nes , Moseley H, MacKie RM. UVA-induced melanocytic lesions. Br $\mathcal{F}$ Dermatol 1987;117:111-5.

Diffey BL. Use of UV-A sunbeds for cosmetic tanning. $\mathrm{Br} \mathcal{F}$ Dermatol 1986;115:67-76.

\section{Treatment of hypercalcaemia of malignancy}

SIR,-Dr A R Morton and others (19 March, p 811) found that a single $60 \mathrm{mg}$ infusion of aminohydroxypropylidene bisphosphonate (APD) was as effective as two $30 \mathrm{mg}$ doses or four $15 \mathrm{mg}$ doses given on consecutive days. Their results accord with the experience of others. ${ }^{1-4}$

One of the aims of the study was to determine the optimal interval between treatments. The average duration of action of APD was unclear from their data-was it a full 30 days, as suggested by fig 1 ? The authors state that further doses of APD were needed occasionally to control recurrent episodes of hypercalcaemia, but it would be helpful to know the proportion of patients who required this, when it was given, and whether there were any differences in the duration of effect between the different regimens.

In a recent (unpublished) study of 55 patients treated with various regimens of APD we found no significant difference in the duration of effect (median about 20 days) or proportion of patients achieving normocalcaemia (about $70 \%$ ) between four $15 \mathrm{mg}$ doses given on consecutive days and single doses of $15 \mathrm{mg}, 25 \mathrm{mg}$, or $45 \mathrm{mg}$. As a result we are now using the $30 \mathrm{mg}$ single dose regimen used by Cantwell and Harris, ${ }^{2}$ reserving higher doses for patients with resistant hypercalcaemia. If the $60 \mathrm{mg}$ dose used by Dr Morton and his colleagues did indeed have a longer duration of action, however, this would be important to doctors treating this syndrome.

STUART H RALSTON

University Department of Medicine, Glasgow Royal Infirmary,

Thiebaud D, Jaeger P, Jacquet AF, Burckhardt P. A single day treatment of tumor induced hypercalcemia by intravenous aminohydroxypropylidene bisphosphor

Cantwell BMJ, Harris AL. Effect of single high dose infusions of aminohydroxypropylidene diphosphonate on hypercalcaemia caused by cancer. Br Med f 1987;294:467-9.

3 Yates AJP, Murray RML, Jerums GJ, Martin TJ. A comparison of single and multiple infusions of 3-amino-1-hydroxypropylidene-1,1-bisphosphonate (APD) in the treatment of hyperca

Coleman RE, Rubens RD. 3-(Amino-1,1-hydroxypropylidene) bisphosphonate (APD) for hypercalcaemia of breast cancer. Brf Cancer 1987;56:465-9.

AUTHOR'S REPLY,-Figure 1 of our study does indeed include data from six patients who were retreated with APD out of 18 who survived for one month after their first treatment. Five were given $30 \mathrm{mg}$ of APD intravenously over two hours at 21 days, and one (a patient with carcinoma of the bronchus) was given this regimen weekly. Our policy is to give $30 \mathrm{mg}$ of APD every three weeks to patients not receiving specific treatment for their tumours when hypercalcaemia recurs in the others.

There was no statistical difference in the duration of normocalcaemia achieved by any of the regimens in this study. While the dose of APD which is effective at restoring normocalcaemia lies between 0.5 and $1.5 \mathrm{mg} / \mathrm{kg},{ }^{1}$ it seems that the duration of infusion of this dose may have a major impact on the response. ${ }^{2}$ Further study is warranted.

A R MORTON

Clinical Sciences Building,

Hope Hospital,

Salford M6 8HD

1 Body J-J, Pot M, Borkowski A, Sculier J-P, Klatersky J. Dose/ response study of aminohydroxypropylidene bisphosphonate in tumor associated hypercalcemia. Am $\mathcal{A}$ Med 1987;82:957-63. treaud D, Jaeger $P$, Jacquet AF, Burckhard P. A single day treatment of tumor induced hypercalcemia by intravenous Mineral Research 1986;1:555-62.

\section{Blood pressure in the very old}

SIR,-Dr Kari Mattila and colleagues (26 March, p 887) showed a negative relation between blood pressure and mortality in an elderly Finnish population. Their conclusion that this was not related to the health of the people studied is surprising and is not supported by the data presented in tables II and III. For example, $50 \%$ of those with the lowest systolic blood pressures were in hospital compared with only $10 \%$ of those with the highest readings. For both systolic and diastolic blood pressure there was a clear trend in the prevalence of dementia, which was highest at low values of blood pressure. Table IV showed that the negative relation between blood pressure and mortality was probably independent of place of residence, but this does not negate the strong evidence of an association between health and blood pressure in their study.

There may be many reasons for an association between low blood pressure and chronic diseases. It may, in part, be due to bias in measurement since unhealthy people are more likely to be familiar with the procedure. Some chronic diseases cause low blood pressure-for example, ischaemic heart disease-and some of the drugs used to treat them reduce blood pressure-for example, diuretics for cardiac failure.

Population based studies of blood pressure and mortality have shown highly variable results. For example, the build and blood pressure study reported a positive relation, ${ }^{1}$ previous studies from Scandinavia have shown $U$ shaped relations, ${ }^{23}$ and the authors' study showed a negative relation. These apparently discrepant findings may be explained on the basis of the association between low blood pressure and chronic diseases discussed above and by the different characteristics of the populations studied. In relatively young and healthy populations low blood pressure rarely indicates chronic disease. In older populations a high prevalence of chronic disease seems to have an 